\title{
A Social Media Analysis of the Gendered Representations of Female and Male Athletes During the 2018 Commonwealth Games
}

\author{
Elaine Chiao Ling Yang, Michelle Hayes, Jinyan Chen, Caroline Riot, and \\ Catheryn Khoo-Lattimore
}

Griffith University, Australia

\begin{abstract}
The contemporary sports culture is characterized as highly masculinized where female athletes are continually marginalized in traditional media. Despite evidence suggesting that media representation of athletes has a meaningful impact on social outcomes and participation rates of women and girls, little is known about gendered representations of athletes on social media and in the context of mega sporting events. This paper examines the gendered representations of athletes on Twitter during the 2018 Commonwealth Games using Framing Theory. A total of 133,338 tweets were analyzed using sentiment and word frequency analyses. Results indicate gender differences concerning athlete representation on Twitter albeit marginal. In particular, the findings reveal that seemingly neutral words (e.g., dedicated, talented and hard-working) could carry gendered connotations. Recommendations are provided to guide stakeholders to advance a more inclusive sports culture through the strategic use of social media during mega sporting events.
\end{abstract}

Keywords: gender, social media, athlete representation, Commonwealth Games, framing theory

Gender equality in sport has received increasing attention in recent years with the emergence of initiatives that promote equality in opportunity, recognition and pay. For instance, large scale event organizations and committees including the International Olympic Committee and the Gold Coast 2018 Commonwealth Games Corporation (GOLDOC) have, respectively, set a target to achieve 50\% female participation in the Olympic Games by 2020 (International Olympic Committee, 2015), and ensure an equal number of medal events for men and women (GOLDOC, 2017). Despite these initiatives, the contemporary sports culture remains highly male-dominated (Dunn, 2018; Hardin \& Greer, 2009).

Sport has been traditionally regarded as a male territory because competition and aggression are deemed as masculine characteristics in contrast to traditional feminine gender roles (Hardin \& Greer, 2009; Koivula, 2001). An assessment of gender appropriateness of sport found that little has changed in the past 50 years, with most sports activities still being rated as masculine (Hardin \& Greer, 2009). Sports media have also typically engaged in gender marking when reporting on women athletes. Gender marking is evident when "an event is identified as a women's event, and the equivalent men's event as the/an event, implying that

The authors are with Griffith Institute for Tourism, Griffith University, Brisbane, Australia. Elaine (e.yang@griffith.edu.au) is corresponding author. This study is funded by Griffith University under the Commonwealth Games Research Projects (CGRP) scheme. 
viewers are getting inferior women's sport, not 'real' (men's) sport" (Wensing \& Bruce, 2003, p. 387). For example, the game of Australian Football played by women is labeled the Australian Football League Women (AFLW), which marks the game as gender-specific. Although there has been a more liberalized attitude toward women's participation in sport, persistent gender stereotyping limits the potential of both young women and men. As a result of dissonance between gender and athletic identities, the dropout rates of girls are two times higher than those of boys by the age of 14, and the dropout age is getting lower (Craike, Symons, \& Zimmermann, 2009; Women's Sports Foundation, 2016).

For women who do participate in sport, they are often subject to sexist representation. For instance, Eugenie Bouchard was asked to perform a twirl to show off her outfit by an oncourt commentator after she had won her match at the 2015 Australian Open (Cormack, 2015). It was at the same tournament in the previous year where she was asked who her ideal date would be. More recently, during a post-match press conference at the 2017 Australian Open, Mirjana Lucic-Baroni, despite having set several records in tennis, was asked whether she and her husband were considering having children any time soon (Salemme, 2017). These gender stereotypes are also evident in social media. For example, Chinese diver He Zi's performance at the 2016 Rio Olympic Games was discredited with her marriage proposal declared as the "perfect score" on Twitter by Australia's official Olympic broadcasting network.

Existing evidence demonstrates that contemporary sports culture remains maledominated with female athletes continuing to be marginalized and sexualized in traditional media. Underpinned by social learning theory (Bandura, 1986) and agenda setting theory (McCombs \& Shaw, 1972), existing literature suggests that changing the representation of athletes in the media will have more meaningful social impacts (e.g., promoting positive images and inspiring young fans, especially girls) than merely increasing women's participation rates (Dunn, 2018; Hardin \& Greer, 2009), because of the power of media in influencing public perception (Eagleman et al., 2014). Although the organizing committees of mega sporting events have committed to increasing women's participation, it is uncertain how this message of equality has reached out to and created an actual impact on the community if the representation of female athletes is continued to be gendered. Furthermore, traditional media is constrained by the amount of information that can be featured within the space and primetime; hence, choices must be made to celebrate some athletes (mostly men) while marginalizing others (mostly women). Conversely, the new media, such as social media has no limitation on the amount of posts, which implies a great opportunity to offer different perspectives and neutralize the masculine sports culture, especially among the younger generation as $99 \%$ of Australians in 15-17 years age group are internet users (Australian Bureau of Statistics, 2016). Nevertheless, limited research has investigated the social media representation of athletes in mega sporting events. Within the handful of studies, most have focused on social media content posted by news and sports organizations (see for example, Litchfield \& Kavanagh, 2019) with little attention to those made by the general public.

Based on the aforementioned gaps, this study investigates the representations of athletes on social media during the 2018 Commonwealth Games from a gender perspective and provides recommendations to promote a more inclusive sports culture. To achieve this objective, the study analyzed tweets with the hashtag, \#GC2018 to assess how female and male athletes were portrayed. Specifically, this study asked: How does the representation of female 
athletes differ from male athletes on social media? The implications of the representations of athletes on social media compared to traditional media were also explored.

\section{Media Representation of Athletes}

\section{Literature Review}

The gendered sports culture has been reproduced and reinforced through media. Multiple studies have demonstrated that male athletes receive significantly more coverage than women athletes despite the latter's increasing participation rates across many sports (Cooky, Messner, \& Hextrum, 2013; Cooky, Messner, \& Musto, 2015). Women only received approximately 5\% to $8 \%$ media coverage, even though they account for $40 \%$ of sport participation (Adams \& Tuggle, 2004; Cooky et al., 2013). Research has demonstrated that the only time women appear to receive an increase in coverage is during mega sporting events such as the Olympic Games and Commonwealth Games (Billings, Angelini, MacArthur, Bissell, \& Smith, 2014; Eagleman, Burch, \& Vooris, 2014). Billings et al. (2014) argued that an increase in attention during megaevents could be explained by national pride where the success of women athletes on national teams is celebrated. While television coverage of women athletes during mega-events is improving when compared to year-round sports coverage, Fink (2015) revealed that "female athletes and women's sport are still woefully underrepresented in all types of media" (p.331). Fink's (2015) review also found that numerous longitudinal studies conducted across different event periods have seen little to no significant increases in the amount of coverage of women athletes (Billings, 2008; Fink, 2015). For instance, Billings (2008) examined telecasts across six Summer and Winter Olympic Games (1996-2006) and identified no significant increase in the coverage for women athletes. Nevertheless, recent research shows that this is slowly changing in Australia as women athletes are beginning to receive more coverage in Australian newspapers (Sherwood, Osborne, Nicholson \& Sherry, 2017).

In addition to media coverage, many researchers have also examined and identified gender differences in the representations of athletes on traditional sports media such as newspapers and television (Adams \& Tuggle, 2004; Cooky et al., 2013; Eagleman, 2015; Godoy-Pressland \& Griggs, 2014). Prior research indicates that when women athletes do receive coverage, they are often trivialized and their achievements in sports are devalued (Biscomb \& Matheson, 2019; Christopherson, Janning, \& McConnell, 2002; Eagleman, 2015; Kane, LaVoi, \& Fink, 2013). Women have typically been regarded as having less athletic skill and less commitment to their sports (Billings \& Eastman, 2002). The language and imagery used to represent women athletes have predominately been sexualized, infantilized, and hyperfeminized (Eagleman, 2015; Godoy-Pressland, 2014; Litchfield, 2015). For instance, coverage has focused on women athletes' physical characteristics while portraying them as sex objects, highlighting femininity in reporting, and comparing their skills and accomplishments to men. The language used to portray women athletes has consisted of "feminine", "pretty", "sexy", "lady/ladies", "beauty", and "graceful" to name a few (Christopherson et al., 2002; Fink, 2015; Jones, Murrell, \& Jackson, 1999; Petty \& Pope, 2019; Thorpe, Toffoletti, \& Bruce, 2017; Wensing \& Bruce, 2003). Nevertheless, recent research suggests that the tone and imagery used to describe female athletes are slowly changing with more women being portrayed as strong, successful and determined (Toffoletti, 2017). In many cases where women athletes were 
not being framed as sexual objects, they were predominantly idealized for their ability to juggle their role as athletes while also being mothers or girlfriends - the socially suitable, fundamental roles of women in relation to men (Cooky et al., 2015). It appears that sports journalists tend to trivialize women athletes by directing focus away from their athletic abilities and focusing on their personal lives (Godoy-Pressland, 2014).

\section{Representation of Athletes on Social Media}

The advent of online media has provided a dynamic space for reporting sports (Litchfield \& Kavanagh, 2019). As technology develops, sports organizations and broadcasting companies have increasingly utilized new media, such as social media platforms to connect with their audience and disseminate information at a more rapid rate (Litchfield \& Kavanagh, 2019). As noted by Hutchins and Mikosza (2010), online media has become an important medium for the promotion and communication of sporting events and athletes. Social media platforms such as Twitter have posed major challenges for sports journalists, who now compete with athletes and sports organizations in reporting news (Sanderson \& Kassing, 2011). The 2012 London Olympics was dubbed the "Twitter Olympics" due to the heightened impact the platform had with athletes and organizers and according to Creedon (2014), "media coverage of the Olympics changed dramatically, eclipsing past newspaper and television Olympic coverage" (p. 712).

Digital journalism appears to be the future of sports media (Creedon, 2014; Sanderson \& Hambrick, 2012; Sheffer \& Schultz, 2010), but few studies have investigated if and how gendered stereotypes in traditional sports media outlets have translated into digital spaces. Of the few studies that have examined the online media coverage of women athletes, Jones (2004) revealed that few women role models were shown on the Australian Broadcast Company's (ABC) website during the coverage of the 2000 Olympic Games. Stereotypical descriptions were reinforced within the little coverage where women were portrayed as emotionally unbalanced and dependent adolescents, whereas men did not receive this infantilized coverage (Jones, 2004). In contrast, a later study revealed that the internet provides sports media an opportunity to create a more accurate depiction of mega-events through more equitable coverage (Eagleman et al., 2014). Eagleman et al. (2014) revealed that few significant gender biases were present in the online coverage of the 2012 Olympic Games in their examination on news websites focusing on six competing nations, including Australia. Given the discrepancy in findings, more research on the debate is warranted.

Although social media is an emerging area of research within the field of sport management and communication (Litchfield, Kavanagh, Osborne, Jones, 2016), only a handful of empirical studies have examined the gendered representation of athletes on social media. Existing studies on social media and athletes have primarily focused on why and how athletes and sports organizations use social media for branding and fan interaction (Clavio \& Kian, 2010; Filo, Lock, \& Karg, 2015; Lebel \& Danylchuk, 2012; Vergeer \& Mulder, 2019). One of the few exceptions is Santarossa, Coyne, Woodruff, and Greenham (2019) which investigated gender disparity in the representation of athletes by ESPN and ESPNW on Instagram around the hashtag, \#BodyIssue. In particular, the study found that male athletes were celebrated more than female athletes. Likewise, Burch, Billings, and Zimmerman (2018) compared Twitter postings surrounding the US men and women soccer teams in the context of the 2014 World 
Cup and identified gender differences in the attributions of success. For instance, women were more likely to be described for their physical poise, playing approach, or as having good luck or fortune, as well as their physical appearance. Litchfield and Kavanagh (2019) examined the representation of male and female athletes on Twitter during the Rio Olympic Games 2016 and revealed the presence of gender marking in the social media space. Although the study has found an increased coverage of female athletes, ambivalence and infantilization are still present in sports reporting. Women athletes continue to be reported as "girls" and "young ladies" whereas male athletes are rarely referred to as "boys" (Fink, 2015; Litchfield \& Kavanagh, 2019; Wensing \& Bruce, 2003). Nevertheless, the sample size of Litchfield and Kavanagh's (2019) study was limited to only 1,455 Twitter posts and focused on social media handles of two sports teams, which were the Australian and Great Britain Olympic teams.

\section{Changing the Representation of Athletes Through Social Media?}

Social media platforms are an interesting point of study as they have the potential to offer different perspectives to those found in traditional media coverage. For instance, Sherwood, Lordanic, Bandaragoda, Sherry, and Alahakoon's (2019) study of the AFLW suggested that the social media community discusses certain topics differently. In the context of the AFLW, the social media community appeared to be focused on the cultural change aspects due to the inception of the league in a traditionally male-dominated sport. Nevertheless, media and sports organizations need to be cautious not to transfer and reinforce the gender stereotypes in online spaces (Sherwood et al., 2019).

Although it is argued that social media has the potential to empower female athletes to change the gendered narratives around women's sports (Toffoletti \& Thorpe, 2018), existing studies suggest that athletes are transferring the sexualized representations to their own social media accounts by using the traditional gender stereotypes to promote themselves due to the impression sports media have on their audiences (Petty \& Pope, 2019). Kane et al. (2013) suggested that women athletes may feel obligated to increase interest in their profiles by following sports media's "sex-selling" strategy. Clavio and Kian (2010) revealed that one of the reasons men were more likely to follow a female athlete on Twitter was due to their physical attractiveness. The sexist representation is observed in new media where women athletes are more likely to post sexually suggestive photos on their personal social media accounts, which may have an impact on perpetuating gender stereotypes and be detrimental to the promotion of women's sports (Geurin-Eagleman \& Burch, 2016).

Prior studies have highlighted the ability social media affords to all sports constituents (i.e., fans, athletes, teams, organizations, sports journalists, and the general public) to shape the representation of athletes (Clavio \& Kian, 2010; Sheffer \& Schultz, 2010). However, social media has simultaneously created a space where unregulated abusive comments about women athletes are evident (Litchfield et al., 2016). Sanderson and Truax (2014) highlighted an increasing development whereby fans criticize athletes via social media with messages manifesting with belittling, sarcasm, threats, and mocking. In their examination of Twitter commentary during the 2015 Wimbledon Championships, Litchfield, Kavanagh, Osborne, and Jones (2018) revealed that Serena Williams became the victim of "gender questioning". Many comments questioned her gender identity and whether she should be playing in the men's competition rather than the women's due to her muscular physique. Comments also questioned 
whether Williams was taking performance-enhancing drugs as a result of her muscular physique. The gender questioning which focused on Williams' physical appearance resonated with gender stereotypes presented in traditional sports media (Litchfield et al., 2018; Toffoletti $\&$ Thorpe, 2018). For instance, women athletes are portrayed as "feminine" and "cute" (Christopherson et al., 2002; Thorpe et al., 2017) but not "muscular".

In contrast to Serena Williams (Litchfield et al., 2018), Maria Sharapova has been portrayed as a "traditionally feminine" woman by sports media, who is "desirable" and "attractive" to sports fans (Litchfield et al., 2016). However, like Williams, Sharapova also became the victim of abuse from social media users during the Wimbledon Championships where public comments primarily focused on her beauty and sexualization, some presented threatening physical and/or sexual contact, while others exhibited emotional ridicule and aggression (Litchfield et al., 2016). Although both women and men athletes can become the victims of online abuse (Browning \& Sanderson, 2012), Litchfield et al.'s $(2016,2018)$ work demonstrates that social media extends the victimization of female athletes by gendered stereotypes originating in traditional media.

The existing literature has suggested the potential of social media in changing the gendered representation of athletes that prevails in traditional media. In particular, social media offers an opportunity for sports organizations, athletes and fans to challenge the prevalent narratives by portraying female athletes in a less sexualized manner and hold them in the same regard as male athletes. Nevertheless, prior studies have also revealed the reproduction of gender stereotypes and the presence of gender-based abuse in the online space. The impact of social media in framing the representation of athletes and shaping public perception is becoming more important due to the widespread use of social media reaching an enormous base of users, plus the ability for the general public to create and disseminate content, which is an ability once exclusive to journalists and news organizations. Despite such importance, few studies have provided a comprehensive investigation of the social media representation of athletes during mega sporting events, and much fewer examined posts made by the general public. To address the research gaps, this study examines the content of Twitter postings by anyone during the 2018 Commonwealth Games to better understand the gendered representations of athletes on social media.

\section{Framing Theory}

Rather than simply identifying the coverage, this study refers to framing theory to explain the issues presented on social media coverage of the 2018 Commonwealth Games. Framing theory (Goffman, 1974) provides an instrumental framework to investigate the narrative in news stories. Specifically, framing theory examines the meanings language journalists use to package news stories through specific frames such as keywords, sentences, sources or visuals (Goffman, 1974; Iyengar, 1991). Framing consists of three distinct stages: frame building, frame setting, and levels of consequences conceived at individual and/or societal levels (D'Angelo, 2002; Scheufele, 2000). Frame building focuses on the selection of what to cover as news, while frame setting consists of the journalists' interpretations of the news content. As the outcome of frame building and setting, audiences may experience altered attitudes about an issue presented by the media. On a societal level, frames may shape decision-making or collective actions (de Vreese, 2005). As previously outlined, sports media need to be cautious 
with how they frame athletes as it can actively shape public opinion (Knight \& Giuliano, 2001). The extent of reach and level of impact is greater than ever with the presence of social media platforms. Furthermore, social media enables any users to create and post content. Each post author may build and set a different frame, but the framing decision is socially constructed to a certain degree and influenced by the dominating discourse and what is deemed acceptable by the society (Oliver \& Johnston, 2000).

Several studies have utilized framing to examine media broadcast commentary of the Olympic Games (e.g., Angelini \& Billings, 2010). More recently the theory has been used to identify the gender differences in newspaper portrayals of male and female gymnasts at the 2012 Olympic Games (Eagleman, 2015). Prior research has extended the application of framing theory to online media. For instance, Romney and Johnson (2019) examined the visual framing of female athletes on sports networks' Instagram accounts and showed that female athletes were framed in gender stereotypes. However, there appears to be a lack of focus on framing and gender representations on Twitter, despite the platform redefining communication among sports constituents. Athletes have used social media to frame their transgressions. For instance, Maria Sharapova utilized social media to frame messages delivered to her fans and the public regarding her doping violation in 2016 (Bell \& Hartman, 2018). Bell and Hartman's (2018) study indicates that framing can be practiced by wider sports constituents rather than being limited to sports journalists. Gender stereotypes of athletes may be reproduced or altered through the frames journalists and public commentators use in their social media posts.

\section{Method}

\section{Data Collection}

The data collection took place during the 2018 Commonwealth Games from 4 April to 15 April 2018. Any publicly available posts on Twitter with the hashtag, \#GC2018 were captured. Twitter was selected as the social media platform for data collection based on the access to publicly available data. Tweets are predominantly public unless users adjust their privacy settings. Competing platforms such as Facebook and Instagram were considered but not selected because Facebook is a semi-public space where posts are usually visible to an articulated list of friends, while Instagram predominantly focuses on visuals (Waterloo, Baumgartner, Peter, \& Valkenburg, 2017). Besides, Twitter is a popular social media with more than 330 million monthly active users (Twitter, 2019). The tweets were harvested using Netlytic, a social network analyzer. In total, 716,914 tweets were harvested and exported to Excel. After removing duplicates and non-English tweets, 133,338 were included in the analysis. Within the dataset, 4210 tweets were posted by news media companies in Australia, organizing committee, destination marketing organizations, sports organizations, and other partner organizations of the event. The remaining tweets were posted by the general public. All tweets were pre-processed to remove meaningless signs and symbols.

In order to process the large and unstructured Twitter data, MongoDB (https://www.mongodb.org/), which is a NoSQL database was employed to string and quarrying data (Stantic \& Pokorny, 2014). NoSQL refers to "not only Structured Query Language (SQL)", which supports not only relational data but also unstructured data, such as the unstructured Twitter data in this study. MongoDB uses a rich declarative query language 
to perform text mining, which facilitates the extraction of useful data through clarifying the purposes in the command (Stantic \& Pokorny, 2014). Once the tweets are processed with useful information, SQL was applied for further analysis in the relational database. To address the research question (i.e., how does the representation of female athletes differ from male athletes on social media during the event?), a series of analyses were conducted over 3 phases.

\section{Phase 1: Analyzing the Gender of Athletes}

The first stage of the analysis involved three steps to separate tweets related to female athletes and those related to males. In step one, a list of gender keywords was used to filter the data. For example, female keywords contained "woman, women, female, girl, girls, she, her", and male keywords included "man, men, male, boy, boys, he, his". In step two, the dataset was analyzed according to the gender of athletes mentioned in the tweets. The names and gender of all athletes who participated in the 2018 Commonwealth Games were extracted from official national sports websites and news articles. Female athlete names $(\mathrm{N}=1905)$ and male athlete names $(\mathrm{N}=2529)$ were imported to phpMyAdmin (https://www.phpmyadmin.net/2, which supports MySQL (Structured Query Language) command to analyze structured data. To decide whether a tweet was related to a certain athlete, the tweet must contain both first and last names of the athlete as there were many athletes sharing the same first or last names.

A list of commands was built on phpMyAdmin to perform the analysis. Step three involved merging the analysis outcomes from steps one and two and removing duplicates. This means that to create the female dataset for example, the female keywords collection (from step 1) and female athletes' collection (from step 2) were merged, and duplicating tweets were eliminated. To ensure that only female-related tweets were included, a list of male keywords was searched within the female dataset and the tweets were deleted if any of the male keywords were mentioned. The same rules applied to the male dataset. Tweets that contained both female and male athlete names or both female and male keywords were omitted. The three stages returned 19,103 tweets contained male athletes' names and male gender keywords and 18,960 tweets with female athletes' names as well as female gender keywords. Figure 1 illustrates the process of analyzing the gender of athletes mentioned in the tweets.

$<$ Insert "Figure 1 - Steps of creating female and male data collection" Here $>$

\section{Phase 2: Sentiment Analysis}

Sentiment analysis was conducted to make an overall comparison between female and male datasets. The sentiment analysis method transfers subjective text to quantitative data and delivers information about the emotional tone of the text. Valence Aware Dictionary for

Sentiment Reasoning (VADER) sentiment analysis method was adopted to assess and compare the sentiment scores of female and male datasets (Hutto \& Gilbert, 2014). VADER relied on the dictionary, which was a pre-set list of positive words with positive scores ( 0 to 4$)$ and negative words with negative scores ( -4 to 0$)$. The output of the sentence polarity is normalized to -1 to +1 for a more effective comparison of results (Becken, Stantic, Chen, Alaei, \& Connolly, 2017). The analysis also took into consideration punctuation, emoticons, and many other heuristics (Chen, Wang, \& Stantic, 2018). 


\section{Phase 3: Word Frequency Analysis}

Word frequency analysis was conducted to analyze the content of the tweets, such as which words were most commonly used in tweets related to female athletes and how did these words differ from those identified in the male dataset. In this phase, four types of analysis were carried out for different purposes.

MapReduce. In order to understand the common topics discussed in the tweets, a MapReduce method was applied to rank the keywords that emerged from the text. MapReduce is a method for efficient grouping and counting a large amount of text. In this analysis, MapReduce consisted of two steps: The first step was "Map" which segmented words by space; the second step was "Reduce" which summed up the same words. A list of words was created based on the weight - the female dataset generated 19,790 different words and the male dataset had 21, 616 words.

Leximancer. Leximancer, a machine-learning content analysis software was also used to identify the key themes and triangulate the results from MapReduce. Leximancer is capable of transforming co-occurring lexicon from text into semantic patterns beyond keyword searching by employing both semantic and relational information extraction (Smith \& Humphreys, 2006). The outcome of Leximancer analysis is presented in a concept map. To generate the concept map, both datasets were imported into Leximancer and analyzed separately. An initial list of concept seeds was generated for each dataset using the default setting. The list was refined by merging synonyms and variations of words that shared the same meaning (Yang, Yang, \& Khoo-Lattimore, 2019) - for example, Commonwealth Games 2018 and CWG2018; win, winning and won, etc. The Leximancer results validated and expanded the results from MapReduce. Nonetheless, the preliminary results from both Map-Reduce and Leximancer indicated no meaningful themes or differences between female and male datasets.

Frequency analysis of gendered words. Because the results from MapReduce and Leximancer did not establish any meaningful themes or differences between female and male datasets, frequency analysis was conducted using a list of gendered words. These words were identified from existing literature (see Table 1) and coding on 200 randomly selected tweets. The manual coding sample is guided by prior research which manually coded between 125 and 400 tweets to develop coding schemes (Jansen, Zhang, Sobel, \& Chowdury, 2009; Lamy et al., 2016). Using computer-generated random numbers, 100 tweets mentioning female athletes and 100 mentioning male athletes were selected. Three researchers coded all selected tweets independently with attention to any words or descriptions that might carry gender connotations. For example, "These Aussie women play some sexy rugby! \#GC2018" was coded as potentially negative and the word, "sexy" was identified for further analysis. The three coders have experience working on gender-related studies and are familiar with the literature. The researchers acknowledged the influence of pre-existing knowledge on coding, but this knowledge also equipped them with the required theoretical sensitivity to identify potential gendered descriptors within the sample. The coding outcomes were compared across the coders and with existing literature. In the case of disagreement, a discussion was held until consensus was reached.

<Insert "Table 1 Gendered Words Identified From Literature” Here> 
A list of 40 words was generated for analysis: attractive, awesome, beauty/beautiful, boy, bronze, calm, champ/champion, classy, convincingly, cool, cute, dedication, family, fantastic, father, feminine, girl/girls, glamor/glamorous, great, hard work, hero, lady, luck, $\mathrm{man} / \mathrm{male} / \mathrm{men}$, mercy/merciful, mother, pretty, prodigy, proud, settle, sex/sexy, simply, sneak, strong, success, talent, unsuccessful, win, woman/women/female, and young. Tweets mentioning any of the 40 words were extracted and analyzed manually to consider the context where the words were used and the gendered connotations. Some tweets mentioned more than one of the 40 words. Altogether, there were 74827 references made to the 40 words. The authors of the posts were also analyzed to identify if there is any difference between those posted by the public and Australian media.

\section{Findings \\ Gender Difference is Marginal but Persists}

The first stage of framing concerns the building of frames, which is what to cover or in this study, who to cover. The findings revealed equal coverage of female and male athletes, and the predominance of sports facts and event specifications (e.g., $100 \mathrm{~m}$ race). Female and male athletes competing at the 2018 Commonwealth Games received a similar amount of attention on social media with $19,103(50.2 \%)$ tweets dedicated to male and 18,960 (49.8\%) to female athletes. Gender difference was observed in the narratives and representations of athletes but on a relatively marginal scale.

The second stage of framing concerns the post authors' interpretations of the content, which were manifested in the narratives and word choices. The sentiment analysis showed statistical difference between tweets mentioning female athletes $(M=0.4401, S D=0.42612)$ and those citing male athletes $(M=0.3969, S D=0.43179) ; t(38061)=9.821, \mathrm{p}<.001$. The sentiment score was 0.04319 higher for female athletes. On the surface, sentiment analysis showed that tweets mentioning female athletes were slightly more positive than males. The analysis, however, did not reflect the gender connotations of the tweets. For example, the sentiment score for this tweet, " 35 years old and a mother of three, only women boxer to win an Olympic Medal in 2012 and here she is oozing so much grit and determination" was 0.8625 , which signified a highly positive score despite the description focused on the athlete's domestic role. Another example was "These Aussie women play some sexy rugby! \#GC2018" which scored 0.7263 .

The initial word frequency analysis using MapReduce and Leximancer method did not indicate any meaningful difference between female and male athletes. The top 20 most frequent words from female and male datasets were selected to compare the differences (see Table 2). To validate and expand the results from MapReduce, two concept maps were generated using Leximancer (see Figure 2). As presented in Table 2 and Figure 2, the most frequently mentioned words were related to sports facts and event specifications. Nevertheless, a closer examination using a list of pre-identified words revealed gender differences in the representation of female and male athletes (see Table 3).

$<$ Insert “Table 2 Top 20 Frequent Words From Female and Male Datasets" Here> 
$<$ Insert "Figure 2 - Concept maps generated using Leximancer" Here>

$<$ Insert "Table 3 Word Frequency Analysis Based on Pre-Determined List of Words" Here>

\section{Female Athletes as Beautiful and Sexy Girls}

As demonstrated in Table 3, female athletes were 2.84 times more likely to be referred to as girls compared to male athletes as boys. Frame setting was present through gendered adjectives such as beauty/beautiful (73:39 or 1.87 times) and sex/sexy (20:10 or 2 times) appeared more frequently in tweets citing female athletes. The analysis shows that sexy was used in conjunction with girls and women but not with boys and men. Some examples of gendered tweets include: "You beautiful golden girls" and "the Aussie women play some sexy rugby". Female athletes were also 2.71 times more likely to be referred to as mothers than males as fathers. Likewise, family was mentioned more frequently in female athlete tweets compared to male tweets.

Nonetheless, in contrast to the literature, no discernible gender difference or opposite outcome was observed in the following words: pretty, cute, feminine, and attractive. Pretty was generally used as an adverb denoting fairly or moderately instead of referring to the physical attractiveness of the athletes. Cute was only mentioned twice in the female dataset and in one instance, it was referred to the win of a female athlete and how cute if her young daughter can use the gold medal as her new teething ring. The analysis of feminine returned no result while attractive was only mentioned in the male athlete dataset.

An analysis of the authors of the posts showed that the Australian news and broadcasting companies generally used gender-neutral descriptions when posting about athletes. There was no mention of sex or sexy in the official media posts and the usage of girls and boys was equal - 38 mentions of girls and 39 of boys. Beauty/beautiful was cited four times but mainly in the context of "beautiful moment", "beautiful routine" and "beautiful act". While there were more references made to mothers in public Tweets when citing female athletes compared to fathers and male athletes, an opposite trend was observed in the official media tweets with two references made to father while no reference made to mother. In both instances, the mentions of father were not directly related to the descriptions of the athletes the tweets were related to a father watching the games with children and a proud grandfather of an athlete watching the competition.

\section{Male Athletes as Young Heroes While Female Athletes as Hardworking and Dedicated}

Frame setting was present in tweets as the results show that male athletes were 1.72 times more likely (or female athletes 0.58 times less likely) to be described as young and 1.21 times (female 0.82 times) as heroes. Some examples of tweets citing hero include: "What a heroic effort from Mark Stewart" and "The man! What a hero". This is especially the case in tweets posted by the Australian media where hero was used more frequently in portraying male athletes. On the other hand, female athletes were about twice as likely to be described by public commentators as hardworking and 5.5 times more likely to be portrayed as dedicated. For instance, the win of one female athlete was described as "the result of her dedication and hard work". A word 
frequency analysis of talent shows that male athletes were 1.56 times (72:46) more likely to be described as talented compared to female athletes - for example, "no words can do justice to his exceptional talent and tenacity". In comparison, prodigy was infrequently mentioned in the data with only 10 mentions associated with female athletes and 11 mentions with males. Interestingly, female athletes were 1.23 times more likely to be portrayed as strong. No reference was made to hard work and dedication in tweets posted by the Australian media while talent was mentioned only once in a tweet on male athletes. The findings imply that for the public commentators on Twitter, the success of female athletes was highly associated with their hard work and dedication rather than talent.

Both women and men were equally portrayed as great (538:509 or female 1.06 times more likely) and successful (151:144 or female 1.05 times more likely) athletes. The word luck was also examined to identify whether the success of athletes from a particular gender group was more likely to be attributed to luck rather than capability. The results show no gender difference as the word frequency of luck was almost equal in both datasets. In most cases, the word, luck was used to wish the athletes "good luck". No difference was observed in the word frequency results for these four words - strong, great, success and luck between tweets posted by the public and those by the Australian media.

\section{Discussion}

\section{Framing Equal Coverage of Female and Male Athletes}

This study sought to investigate the gender difference in the representation of female and male athletes on social media during the 2018 Commonwealth Games. The current study highlighted that Twitter users provided a relatively equal amount of coverage of female and male athletes. This is in contrast to previous findings that suggest reporting of female athletes is dramatically underrepresented in sports media (e.g., Cooky et al., 2013; Cooky et al., 2015). The study lends support to a scarce amount of research that suggests digital spaces (e.g., social media) are linked to increases in coverage of female athletes (Litchfield \& Kavanagh, 2019). Further, in terms of the selection of coverage and frame building, Twitter postings focused more on sports facts and event specifications, which could be explained by the equal medal opportunities afforded to female and male athletes at the 2018 Commonwealth Games. Equal numbers of medal opportunities for female and male athletes may have influenced the coverage.

\section{(Re)Setting the Representations of Athletes}

Although improvements on frame building were evident, the study lends support to existing literature regarding the gendered representation of female athletes who have been consistently described as beautiful and sexy, and associated with traditional female roles in family as mothers (Christopherson et al., 2002; Fink, 2015; Wensing \& Bruce, 2003). Nevertheless, the findings of this study reveal that some of the gendered words (e.g., pretty, feminine and attractive) identified in the traditional media (Christopherson et al., 2002; Jones et al, 1999; Wensing \&, Bruce, 2003) did not apply to the current study in a social media context. Similarly, the portrayal of female athletes as 'strong' is in contrast to prior research which suggests that strong is a long-standing descriptor for male athletes emanating in the 1960s (Wanneberg, 2011) and included in sports coverage in the 2004 and 2008 Olympic Games (Petca, Bivolaru, 
$\&$ Graf, 2013). The change in narratives suggests that Twitter may allow users to alter the frame setting previously used to portray female athletes.

\section{Theoretical Contributions}

The findings of the current study contribute to the first two stages of framing theory in a social media context. The use of Twitter suggested that the concept of framing can be transferred to social media contexts and adopted by all users and sports constituents, not just sports journalists. This finding addresses a gap in the literature where previous research has predominately focused on sports journalists (e.g., Angelini \& Billings, 2010; Eagleman, 2015), or athletes' ability to frame transgressions (e.g., Bell \& Hartman, 2018). As previously noted, Twitter afforded its users to equally cover female and male athletes. This could have been influenced by some of Twitter users' preferences (e.g., users more interested in watching female competitions) (Sherwood et al., 2019). Twitter is accessible to many people and gives them a voice, which presents an opportunity for diverse voices and alternative portrayals of female athletes. Nevertheless, Twitter users may have also been influenced by the frames previously used to portray female athletes by sports and traditional media. For instance, gender differences in frame setting were evident albeit at a marginal scale between female and male athletes in this study. This highlights important implications for frame setting and sports journalists as they could influence the language used by other Twitter users (Knight \& Giuliano, 2001). For a considerable amount of time sports journalists have framed female athletes using gendered stereotypes and infantilizing language (Eagleman, 2015; GodoyPressland, 2014; Litchfield, 2015). Although improvements were evident in this study, gendered stereotypes appear to have transmitted to the Twitter environment.

Furthermore, the study adds to existing knowledge by showing that seemingly neutral words such as talent, hard work and dedication could carry a gendered connotation - the success of male athletes was associated with talent while female's success was attributed to hard work and dedication. This is problematic because it predisposes the notion that men are natural athletes while women are not. As a consequence, it primes the broader community which may also include athletes, to agree with these gendered qualities. Eventually, male athletes would bank on confidence rather than competence while female athletes develop the imposter syndrome and take on unnecessary physical and mental stress. This finding on female athletes having to work hard versus male athletes having talent is uncannily similar to research in management and leadership, where men have been assumed to be natural leaders while women spend much more time proving themselves in the boardroom, or emulating and displaying assumed male leadership qualities to the extent that they "out-male" their male colleagues in masculinity (Rigg \& Sparrow, 1994).

\section{Practical Implications}

The findings point to internalized stereotypes about women's sporting abilities (e.g., they do not have the talent as men do), which has the potential to negatively influence the likelihood of women to pursue sports as a career compared to men. This very insight can be used to influence sports recruitment and sports career development communication strategies. The outcomes of the study can be used to raise awareness among male and female athletes about the potential social impacts of their engagement with and self-representation on social media 
and how they can advance new ways of thinking about gender and sports. The findings can be translated into a gender mainstreaming curriculum for sports education and training, but also used in short professional development courses for journalists and media members.

The opportunity to revise existing approaches to sports education and training in this space has the potential to also advance male and female journalists and sports writers' framing of athletes in print-media coverage. While the focus of the research is on the strategic use of social media during mega sporting events, the findings are also relevant to traditional media outlets. In the context of major stories surrounding sports events, new media outlets tend to focus solely on individual athletes and may operate with different standards and practices than those in traditional sports media (Whiteside, Yu, \& Hardin, 2012). A lack of depth in the level of critical analysis surrounding topics of discussion in new media presents an opportunity for traditional media to re-emphasize its contribution to critical analysis, commentary, and narratives relating to major sports events and gendered perspectives. As Vann (2014) points out, social media is now an integral part of modern sports broadcasting combining old and new media with particular implications for women's sports due to this convergence. For example, while organizations can work to address some of the issues women's sports face from a lack of traditional broadcast coverage, there is also an opportunity to rethink how traditional media can extend its influence through reframing work.

Based on the findings, recommendations are proposed to guide stakeholders towards advancing a more inclusive sports culture through the strategic use of social media during mega sporting events. First, sports organizations are encouraged to lead change in the representation of female athletes in social media by promoting positive images and using language to capture athletic characteristics not bound by gender (e.g., competitiveness, robustness, athleticism). While news and broadcasting companies generally use gender-neutral descriptions when posting about athletes, gender representation can be advanced through proactive and transparent dialog among sports organizations, large scale event organizations, and media groups during partnership negotiation processes around the promotion and communication of athletes in sports events. Second, there is a need for development and education work among athletes and player's associations around athlete brand awareness and personal identity, and significant work with key stakeholders around removing gender marking in sport (e.g., avoiding the verbal and visual representation of male athletes and men's sports as the norm with women athletes and competitions as secondary). Sports organizations could play an important role to foster engagement with key stakeholders (including athletes) around the appropriate language, imagery, and tone to describe female athletes in social media, with the anticipated outcome of stakeholder's being champions of positive perceptions of women's athletic abilities comparable to their male counterparts.

\section{Limitations and Future Directions}

While the findings of this study support the persisting sexist and gendered representations of female athletes, such representations were observed on a marginal scale. It is not known to what extent this indicates actual progress in the gendered representation of athletes. Twitter is about getting information out first and quicker thus the narrative is less emphasized (Sanderson \& Hambrick, 2012). The limitation of the length of tweets (i.e., 140 characters) has required the authors of the posts to be succinct and focus on the key messages, which as observed in our 
data, were mainly reporting the competition outcomes. As such, one positive implication derived from this study was that the brief nature of social media posting has made the content more focused and hence, neutralized the representation of female and male athletes at mega sporting events. Nevertheless, such effects may have changed as Twitter has extended the content from 140 to 280 characters. Further research is warranted to examine how this change in content length affects the representation of athletes as more length means more space for descriptions and narratives. Another research avenue is to conduct a similar study on Instagram and/or Facebook where posts are not limited to word count. In particular, research analyzing images that complement the social media posts will add valuable insights to the framing and representation of female and male athletes from a visual perspective. Likewise, an investigation of the effect of authors' gender on the post content will advance the existing understanding of the gendered framing of athletes.

Furthermore, the marginal gender differences could be explained by the limitation of the data. A large volume of text data was harvested using a generic hashtag for the event, \#gc2018 rather than any specific gendered hashtag such as \#metoo which was related to sexual harassment, or \#SerenaWilliams which was used to discuss sexism during the 2018 US Open Tennis Championships. While the extracted data were appropriate for the purpose of this study, which was to examine the representation of athletes at the Commonwealth Games, the amount of noise (i.e., irrelevant text) increased proportionately with the volume of data. More studies are warranted to focus on recent sexist incidents and the related comments on social media to provide a more holistic understanding of the effect of social media in portraying and shaping the public perception of athletes. The third stage of framing, level of consequences, was not covered in the current study due to the focus on gendered representations of athletes via Twitter. The findings of the study suggest that the continued use of descriptors with gendered connotations could be of consequence to the general public and the perceptions of female athletes. However, more research is needed to understand the level of consequences in terms of the perceptions of those reading, retweeting and responding to Twitter postings of the event and whether it influences their perceptions of female athletes.

The lack of gendered insights from automated analyses, such as sentiment analysis and machine-generated word frequency analysis implies the possible gender bias that is inherent in existing programming. This assumption was supported by the manual analysis where gender differences were identified. For example, girl, mother, and family were mentioned more frequently in the female athlete dataset compared to boy, father, and family in the male dataset, but the mention of girl, mother or family does not give the tweet a negative sentiment score. Future research is encouraged to build a specialized gender dictionary similar to that of sentiment analysis for big data analysis but with a gender score. For instance, if the data contains any gendered or sexist word, it will return a negative score. Furthermore, this study analyzed and compared datasets containing tweets on female athletes and those on male athletes to identify any gender differences; tweets mentioning both female and male athletes were omitted due to the limitation of the text mining tools for effective comparison. Further research is warranted to investigate the omitted sub-sample. For instance, an athlete may be referred to as the wife, girlfriend, husband or boyfriend of another athlete - in this case, both female and male athletes are mentioned within the same tweet. An analysis of such descriptions will add insights into how gender stereotypes or norms are framed and reinforced through the 
representations of athletes in domestic roles.

\section{Conclusion}

Theoretically, this study has advanced the understanding of the gendered representation of athletes in online media space. The study has built the groundwork for future research to deconstruct the underlying meanings of words, especially seemingly neutral words used to portray athletes. The focus on social media distinguishes this study from existing research, which has mainly assessed gendered representation in traditional media outlets (e.g., print and televised news media). As mentioned in the introduction, social media, in contrast to traditional media which is highly constrained in content space and primetime, presents a great opportunity to neutralize the masculine sports culture, especially among young Australians. Nevertheless, limited research has investigated the social media representation of athletes at mega sporting events and even less on social media content posted by the general public. As such, this study has addressed this gap by advancing knowledge on the gender representation of athletes made by media organizations and public commentators on Twitter during the 2018 Commonwealth Games.

This study provided insights into the social media representation of female and male athletes at mega sporting events with a specific focus on gender representation. Overall, the analysis of common topics discussed in tweets relevant to gender representations of athletes and the descriptions of those athletes work to produce useful findings for researchers, media organizations, and sport practitioners working in high-performance sport and mega-events. While female and male athletes receive a similar amount of attention on social media, marginal differences are observed in the gendered narratives and representations of athletes. News and broadcasting companies generally use gender-neutral descriptions in posts about athletes, yet male athletes are often depicted as young heroes while females are described as hardworking and dedicated by public commentators. Interestingly, the use of the term "girls" when referring to female athletes (compared to "boy" for male athletes) and "sex/sexy" persist in a more frequent manner for female athletes. These findings indicate that more work is needed to better align the representations of female athletes with the overarching objective of sport custodians to reduce the marginalization and sexualization of female athletes on social media and produce a meaningful impact on social outcomes and participation rates of women and girls.

\section{References}

Adams, T., \& Tuggle, C. A. (2004). ESPN's SportsCenter and coverage of women's athletics: "It's a boys' club". Mass Communication \& Society, 7(2), 237-248. doi: $10.1207 / \mathrm{s} 15327825 \mathrm{mcs} 0702 \_6$

Angelini, J. R., \& Billings, A. C. (2010). An agenda that sets the frames: Gender, language, and NBC's Americanized Olympic telecast. Journal of Language and Social Psychology, 29(3), 363-385. doi: 10.1177/0261927X10368831

Australian Bureau of Statistics. (2016). Household use of information technology, Australia, 2014-15 [Statistical release]. Retrieved from http://www.abs.gov.au/AUSSTATS/abs@.nsf/Lookup/8146.0Main+Features1201415?OpenDocument 
Bandura, A. (1986). Social foundations of thought and action: A social cognitive theory. Englewood Cliffs, NJ: Prentice Hall.

Becken, S., Stantic, B., Chen, J., Alaei, A. R., \& Connolly, R. M. (2017). Monitoring the environment and human sentiment on the Great Barrier Reef: Assessing the potential of collective sensing. Journal of Environmental Management, 203(1), 87-97. doi: 10.1016/j.jenvman.2017.07.007

Bell, T. R., \& Hartman, K. L. (2018). Stealing thunder through social media: The framing of Maria Sharapova's drug suspension. International Journal of Sport Communication, 11(3), 369-388. doi: 10.1123/ijsc.2018-0079

Billings, A. C. (2008). Olympic media: Inside the biggest show on television. London, England: Routledge.

Billings, A. C., \& Eastman, S. T. (2002). Selective representation of gender, ethnicity, and nationality in American television coverage of the 2000 Summer Olympics. International Review for the Sociology of Sport, 37(3-4), 351-370. doi: $10.1177 / 101269020203700302$

Billings, A. C., Angelini, J. R., MacArthur, P. J., Bissell, K., \& Smith, L. R. (2014). (Re)Calling London: The gender frame agenda within NBC's primetime broadcast of the 2012 Olympiad. Journalism \& Mass Communication Quarterly, 91(1), 38-58. doi: $10.1177 / 1077699013514416$

Biscomb, K., \& Matheson, H. (2019). Are the times changing enough? Print media trends across four decades. International Review for the Sociology of Sport, 54(3), 259-281. doi: $10.1177 / 1012690217716574$

Browning, B., \& Sanderson, J. (2012). The positives and negatives of Twitter: Exploring how student-athletes use Twitter and respond to critical tweets. International Journal of Sport Communication, 5(4), 503-521. doi: 10.1123/ijsc.5.4.503

Burch, L. M., Billings, A. C., \& Zimmerman, M. H. (2018). Comparing American soccer dialogues: Social media commentary surrounding the 2014 US men's and 2015 US women's World Cup teams. Sport in Society,21(7), 1047-1062. doi: 10.1080/17430437.2017.1284811

Chen, J., Wang, S., \& Stantic, B. (2018). Connecting social media data with observed hybrid data for environment monitoring. In M. Ivanović, C. Bădică, J. Dix, Z. Jovanović, M. Malgeri, \& M. Savić (Eds.), Studies in Computational Intelligence Series: Vol. 737. Intelligent Distributed Computing XI (pp. 125-135). doi: 10.1007/978-3-319-66379$1 \_12$

Christopherson, N., Janning, M., \& McConnell, E. D. (2002). Two kicks forward, one kick back: A content analysis of media discourses on the 1999 Women's World Cup soccer championship. Sociology of Sport Journal, 19(2), 170-188. doi: 10.1123/ssj.19.2.170

Clavio, G., \& Kian, T. M. (2010). Uses and gratifications of a retired female athlete's Twitter followers. International Journal of Sport Communication, 3(4), 485-500. doi: 10.1123/ijsc.3.4.485

Cooky, C., Messner, M. A., \& Hextrum, R. H. (2013). Women play sport, but not on TV. Communication \& Sport, 1(3), 203-230. doi: 10.1177/2167479513476947

Cooky, C., Messner, M. A., \& Musto, M. (2015). "It's dude time!” A quarter century of excluding women's sports in televised news and highlight shows. Communication \& 
Sport, 3(3), 261-287. doi: 10.1177/2167479515588761

Cormack, L. (2015, January 22). Eugenie Bouchard asked to 'give us a twirl' in on-court interview. The Sydney Morning Herald. Retrieved from https://www.smh.com.au

Craike, M. J., Symons, C., \& Zimmermann, J. A. M. (2009). Why do young women drop out of sport and physical activity? A social ecological approach. Annals of Leisure Research, 12(2), 148-172. doi: 10.1080/11745398.2009.9686816

Creedon, P. (2014). Women, social media, and sport: Global digital communication weaves a web. Television and New Media, 15(8), 711-716. doi: 10.1177/1527476414530476

D'angelo, P. (2002). News framing as a multi-paradigmatic research program: A response to Entman. Journal of Communication,52(4), 870-888. doi: 10.1111/j.14602466.2002.tb02578.x

De Vreese, C. H. (2005). News framing: Theory and typology. Information Design Journal \& Document Design, 13(1), 51-62.

Dunn, C. (2018). Media coverage of women's sport: Personal reflections. In L. Mansfield, J. Caudwell, B. Wheaton, \& B. Watson (Eds.), The Palgrave handbook of feminism and sport, leisure and physical education (pp. 841-853). London, England: Palgrave Macmillan.

Eagleman, A., Burch, L. M., \& Vooris, R. (2014). A unified version of London 2012: Newmedia coverage of gender, nationality, and sport for Olympics consumers in six countries. Journal of Sport Management, 28(4), 457-470. doi: 10.1123/jsm.2013-0151

Eagleman, A. N. (2015). Constructing gender differences: Newspaper portrayals of male and female gymnasts at the 2012 Olympic Games. Sport in Society, 18(2), 234-247. doi: $10.1080 / 17430437.2013 .854509$

Filo, K., Lock, D., \& Karg, A. (2015). Sport and social media research: A review. Sport Management Review, 18(2), 166-181. doi: 10.1016/j.smr.2014.11.001

Fink, J. S. (2015). Female athletes, women's sport, and the sport media commercial complex: Have we really “come a long way, baby"? Sport Management Review, 18(3), 331-342. doi: 10.1016/j.smr.2014.05.001

Franciscus N., Ren X., Stantic B. (2018). Beyond word-cloud: A graph model derived from beliefs. In N. Nguyen, D. Hoang, T. P. Hong, H. Pham, \& B. Trawiński (Eds), Lecture Notes in Computer Science: Vol. 10752. Intelligent Information and Database Systems (pp. 81-90). doi: 10.1007/978-3-319-75420-8_8

Franciscus, N., Ren, X., \& Stantic, B. (2019). Dependency graph for short text extraction and summarization. Journal of Information and Telecommunication, 3(4), 413-429. doi: 10.1080/24751839.2019.1598771

Geurin-Eagleman, A. N., \& Burch, L. M. (2016). Communicating via photographs: A gendered analysis of Olympic athletes' visual self-presentation on Instagram. Sport Management Review, 19(2), 133-145. doi: 10.1016/j.smr.2015.03.002

Godoy-Pressland, A. (2014). The weekend as a male entity: How Sunday newspaper sports reporting centres around male activities, interests and language (2008 and 2009). Leisure Studies, 33(2), 148-163. doi: 10.1080/02614367.2013.833286

Godoy-Pressland, A., \& Griggs, G. (2014). The photographic representation of female athletes in the British print media during the London 2012 Olympic Games. Sport in Society, 17(6), 808-823. doi: 10.1080/17430437.2014.882908 
Goffman, E. (1974). Frame analysis: An essay on the organization of experience. New York, NY: Harper \& Row.

GOLDOC. (2017). Celebrating women in sport [Article]. Retrieved from https://www.gc2018.com/article/celebrating-women-sport

Hardin, M., \& Greer, J. D. (2009). The influence of gender-role socialization, media use and sports participation on perceptions of gender-appropriate sports. Journal of Sport Behavior, 32(2), 207-226.

Hutchins, B., \& Mikosza, J. (2010). The web 2.0 Olympics: Athlete blogging, social networking and policy contradictions at the 2008 Beijing Games. Convergence, 16(3), 279-297. doi: $10.1177 / 1354856510367618$

Hutto, C. J., \& Gilbert, E. (2014). VADER: A parsimonious rule-based model for sentiment analysis of social media text. Proceedings of the Eighth International AAAI Conference on Weblogs and Social Media, 216-225. Retrieved from https://www.aaai.org/ocs/index.php/ICWSM/ICWSM14/paper/viewPaper/8109

International Olympic Committee. (2015). Promotion of women in sport through time. Retrieved from https://www.olympic.org/women-in-sport/background

Iyengar, S. (1991). Is anyone responsible? Chicago, IL: University of Chicago Press.

Jansen, B. J., Zhang, M., Sobel, K., \& Chowdury, A. (2009). Twitter power: Tweets as electronic word of mouth. Journal of the American Society for Information Science and Technology, 60(11), 2169-2188. doi: 10.1002/asi.21149

Jones, D. (2004). Half the story? Olympic women on ABC news online. Media International Australia Incorporating Culture and Policy, 110(1), 132-146. doi: 10.1177/1329878X0411000114

Jones, R., Murrell, A. J., \& Jackson, J. (1999). Pretty versus powerful in the sports pages: Print media coverage of US women's Olympic gold medal winning teams. Journal of Sport and Social Issues, 23(2), 183-192. doi: 10.1177/0193723599232005

Kane, M. J., LaVoi, N. M., \& Fink, J. S. (2013). Exploring elite female athletes' interpretations of sport media images: A window into the construction of social identity and "selling sex" in women's sports. Communication \& Sport, 1(3), 269-298. doi: $10.1177 / 2167479512473585$

Knight, J. L., \& Giuliano, T. A. (2001). He's a Laker; she's a "looker": The consequences of gender-stereotypical portrayals of male and female athletes by the print media. Sex Roles, 45(3-4), 217-229. doi: 10.1023/A:1013553811620

Koivula, N. (2001). Perceived characteristics of sports categorized as gender-neutral, feminine and masculine. Journal of Sport Behavior, 24(4), 377-393.

Lamy, F. R., Daniulaityte, R., Sheth, A., Nahhas, R. W., Martins, S. S., Boyer, E. W., \& Carlson, R. G. (2016). "Those edibles hit hard": Exploration of Twitter data on cannabis edibles in the U.S. Drug and Alcohol Dependence, 164, 64-70. doi: 10.1016/j.drugalcdep.2016.04.029

Lebel, K., \& Danylchuk, K. (2012). How tweet it is: A gendered analysis of professional tennis players' self-presentation on Twitter. International Journal of Sport Communication, 5(4), 461-480. doi: 10.1123/ijsc.5.4.461

Litchfield, C. (2015). A media analysis of Ellyse Perry and the rise? of women's sport in Australia. Journal of Sporting Cultures and Identities, 6(1), 11-22. 
Litchfield, C., \& Kavanagh, E. (2019). Twitter, Team GB and the Australian Olympic Team: Representations of gender in social media spaces. Sport in Society, 22(7), 1148-1164. doi: 10.1080/17430437.2018.1504775

Litchfield, C., Kavanagh, E. J., Osborne, J., \& Jones, I. (2016). Virtual maltreatment: Sexualisation and social media abuse in sport. Psychology of Women Section Review, 18(2), 36-47.

Litchfield, C., Kavanagh, E., Osborne, J., \& Jones, I. (2018). Social media and the politics of gender, race and identity: The case of Serena Williams. European Journal for Sport and Society, 15(2), 154-170. doi: 10.1080/16138171.2018.1452870

McCombs, M. E., \& Shaw, D. L. (1972). The agenda-setting function of mass media. Public Opinion Quarterly, 36(2), 176-187. doi: 10.1086/267990

Oliver, P., \& Johnston, H. (2000). What a good idea! Ideologies and frames in social movement research.Mobilization: An International Quarterly,5(1), 37-54. doi: 10.17813/maiq.5.1.g54k222086346251

Petca, A. R., Bivolaru, E., \& Graf, T. A. (2013). Gender stereotypes in the Olympic Games media? A cross-cultural panel study of online visuals from Brazil, Germany and the United States. Sport in Society, 16(5), 611-630. doi: 10.1080/17430437.2012.690412

Petty, K., \& Pope, S. (2019). A new age for media coverage of women's sport? An analysis of English media coverage of the 2015 FIFA Women's World Cup. Sociology, 53(3), 486502. doi: 10.1177/0038038518797505

Rigg, C., \& Sparrow, J. (1994). Gender, diversity and working styles. Women in Management Review, 9(1), 9-16. doi: 10.1108/09649429410050971

Romney, M., \& Johnson, R. G. (2019). The ball game is for the boys: The visual framing of female athletes on national sports networks' Instagram accounts. Communication \& Sport. Advance online publication. doi: 10.1177/2167479519836731

Salemme, K. (2017, January 24). When journalists cross the line in post-match interviews. News.com.au. Retrieved from https://www.news.com.au/

Sanderson, J., \& Hambrick, M. E. (2012). Covering the scandal in 140 characters: A case study of Twitter's role in coverage of the Penn State saga. International Journal of Sport Communication, 5(3), 384-402. doi: 10.1123/ijsc.5.3.384

Sanderson, J., \& Kassing, J. W. (2011). Tweets and blogs: Transformative, adversarial, and integrative developments in sports media. In A.C. Billings (Ed.), Sports media: Transformation, integration, consumption (pp. 114-127). New York, NY: Routledge.

Sanderson, J., \& Truax, C. (2014). "I hate you man!”: Exploring maladaptive parasocial interaction expressions to college athletes via Twitter. Journal of Issues in Intercollegiate Athletics, 7, 333-351.

Santarossa, S., Coyne, P., Woodruff, S. J., \& Greenham, C. G. (2019). \#BodyIssue and Instagram: A gender disparity in conversation, coverage, and content in ESPN the magazine. International Journal of Sport Communication, 12(3), 336-353. doi: 10.1123/ijsc.2018-0159

Scheufele, D. A. (2000). Agenda-setting, priming, and framing revisited: Another look at cognitive effects of political communication. Mass Communication \& Society, 3(2-3), 297-316. doi: 10.1207/S15327825MCS0323_07

Sheffer, M. L., \& Schultz, B. (2010). Paradigm shift or passing fad? Twitter and sports 
journalism. International Journal of Sport Communication, 3(4), 472-484. doi: 10.1123/ijsc.3.4.472

Sherwood, M., Lordanic, M., Bandaragoda, T., Sherry, E., \& Alahakoon, D. (2019). A new league, new coverage? Comparing tweets and media coverage from the first season of AFLW. Media International Australia, 172(1), 114-130. doi: $10.1177 / 1329878 X 19852495$

Sherwood, M., Osborne, A., Nicholson, M., \& Sherry, E. (2017). Newswork, news values, and audience considerations: Factors that facilitate media coverage of women's sports. Communication \& Sport, 5(6), 647-668. doi: 10.1177/2167479516645535

Smith, A. E., \& Humphreys, M. S. (2006). Evaluation of unsupervised semantic mapping of natural language with Leximancer concept mapping. Behavior Research Methods, 38(2), 262-279. doi: 10.3758/BF03192778

Stantic, B., \& Pokorny, J. (2014). Opportunities in big data management and processing. In H. M. Haav, A. Kalja, \& T. Robal (Eds.), Databases and information systems viii (pp. 1526). Amsterdam, Netherlands: IOS Press.

Thorpe, H., Toffoletti, K., \& Bruce, T. (2017). Sportswomen and social media: Bringing thirdwave feminism, postfeminism, and neoliberal feminism into conversation. Journal of Sport and Social Issues, 41(5), 359-383. doi: 10.1177/0193723517730808

Toffoletti, K. (2017). Sexy women sports fans: Femininity, sexuality, and the global sport spectacle. Feminist Media Studies, 17(3), 457-472. doi: 10.1080/14680777.2016.1234499

Toffoletti, K., \& Thorpe, H. (2018). Female athletes' self-representation on social media: A feminist analysis of neoliberal marketing strategies in "economies of visibility". Feminism \& Psychology, 28(1), 11-31. doi: 10.1177/0959353517726705

Twitter. (2019). Q1 2019 earnings report [Report]. Retrieved from https://s22.q4cdn.com/826641620/files/doc_financials/2019/q1/Q1-2019-SlidePresentation.pdf

Vann, P. (2014). Changing the game: The role of social media in overcoming old media's attention deficit toward women's sport. Journal of Broadcasting \& Electronic Media, 58(3), 438-455. doi: 10.1080/08838151.2014.935850

Vergeer, M., \& Mulder, L. (2019). Football players' popularity on Twitter explained: Performance on the pitch or performance on Twitter? International Journal of Sport Communication, 12(3), 376-396. doi: 10.1123/ijsc.2018-0171

Wanneberg, P. L. (2011). The sexualization of sport: A gender analysis of Swedish elite sport from 1967 to the present day. European Journal of Women's Studies, 18(3), 265-278. doi: $10.1177 / 1350506811406075$

Waterloo, S. F., Baumgartner, S. E., Peter, J., \& Valkenburg, P. M. (2017). Norms of online expressions of emotion: Comparing Facebook, Twitter, Instagram, and WhatsApp. New Media \& Society, 20(5), 1813-1831. doi: 10.1177/1461444817707349

Wensing, E. H., \& Bruce, T. (2003). Bending the rules: Media representations of gender during an international sporting event. International Review for the Sociology of Sport, 38(4), 387-396. doi: 10.1177/1012690203384001

Whiteside, E., Yu, N., \& Hardin, M. (2012). The new "toy department"? A case study on differences in sports coverage between traditional and new media. Journal of Sports 
Media, 7(1), 23-38. doi: 10.1353/jsm.2012.0000

Women's Sport Foundation. (2016). Do you know the factors influencing girls' participation in sports? Retrieved from https://www.womenssportsfoundation.org/support-us/do-youknow-the-factors-influencing-girls-participation-in-sports/

Yang, M. J. H., Yang, E. C. L., \& Khoo-Lattimore, C. (2019). Host-children of tourism destinations: Systematic quantitative literature review. Tourism Recreation Research. Advance online publication. doi: 10.1080/02508281.2019.1662213 
Table 1 Gendered Words Identified From Literature

\begin{tabular}{ll}
\hline Gendered Words & Sources \\
\hline Girl & Litchfield and Kavanagh (2019); Wensing and Bruce (2003) \\
Young Lady & Wensing and Bruce (2003) \\
Beauty/beautiful & Fink (2015); Wensing and Bruce (2003) \\
Attractive & Wensing and Bruce (2003) \\
Glamor & Fink (2015); \\
Lady & Petty and Pope (2019) \\
Feminine & Christopherson, Janning, and McConnell (2002) \\
Sexy & Christopherson et al. (2002) \\
Mother & Christopherson et al. (2002) \\
Family & Christopherson et al. (2002) \\
Cute & Thorpe, Toffoletti, and Bruce (2017) \\
Classy & Kane, LaVoi, and Fink (2013) \\
Pretty & Jones, Murrell, and Jackson (1999) \\
\hline
\end{tabular}

Table 2 Top 20 Frequent Words From Female and Male Datasets

\begin{tabular}{lclc}
\hline Male Dataset & Weight & Female Dataset & Weight \\
\hline \#GC2018 & 18392 & \#GC2018 & 18978 \\
Men & 10451 & Women & 10938 \\
Gold & 6780 & Gold & 8224 \\
Medal & 5142 & India & 4688 \\
India & 4167 & Medal & 4533 \\
Final & 3394 & Congratulations & 3033 \\
Bronze & 2667 & Silver & 2741 \\
\#Cwg2018 & 2608 & Final & 2740 \\
Congratulations & 2377 & \#Cwg2018 & 2472 \\
Wins & 2342 & Games & 2433 \\
Games & 2331 & Wins & 2426 \\
Men's & 2297 & Event & 2425 \\
Live & 1999 & Team & 2293 \\
Event & 1932 & Women's & 2269 \\
Commonwealth & 1857 & \#CommonwealthGames2018 & 2026 \\
\#CommonwealthGames2018 & 1824 & Winning & 1974 \\
Silver & 1809 & Commonwealth & 1921 \\
Kg & 1777 & Bronze & 1826 \\
Team & 1768 & Win & 1697 \\
Winning & 1499 & Live & 1627 \\
\hline
\end{tabular}


Table 3 Word Frequency Analysis Based on Pre-Determined List of Words

\begin{tabular}{|c|c|c|c|c|}
\hline $\begin{array}{l}\text { List of Gendered } \\
\text { Words }\end{array}$ & $\begin{array}{c}\text { Tweets on } \\
\text { Female } \\
\text { Athletes }\end{array}$ & $\begin{array}{l}\text { Tweets on } \\
\text { Male } \\
\text { Athletes }\end{array}$ & $\begin{array}{c}\text { Gender } \\
\text { Difference* }\end{array}$ & $\begin{array}{c}\text { Gender } \\
\text { Difference } \\
\text { (Ratio) }^{* *}\end{array}$ \\
\hline Dedication & 66 & 12 & 54 & 5.50 \\
\hline Mercy/Merciful & 5 & 1 & 4 & 5.00 \\
\hline Classy & 7 & 2 & 5 & 3.50 \\
\hline Girl or Boy & 1940 & 684 & 1256 & 2.84 \\
\hline Mother or Father & 19 & 7 & 19 & 2.71 \\
\hline Sneak & 11 & 5 & 6 & 2.20 \\
\hline Sex/Sexy & 20 & 10 & 10 & 2.00 \\
\hline Unsuccessful & 2 & 1 & 1 & 2.00 \\
\hline Hard Work & 21 & 11 & 10 & 1.91 \\
\hline Beauty/Beautiful & 73 & 39 & 34 & 1.87 \\
\hline Awesome & 112 & 68 & 44 & 1.65 \\
\hline Fantastic & 131 & 82 & 49 & 1.60 \\
\hline Silver & 3186 & 2088 & 1098 & 1.53 \\
\hline Proud & 1442 & 954 & 488 & 1.51 \\
\hline Family & 51 & 36 & 15 & 1.42 \\
\hline Champ/Champion & 525 & 392 & 133 & 1.34 \\
\hline Gold & 9514 & 7579 & 1935 & 1.26 \\
\hline Strong & 117 & 95 & 22 & 1.23 \\
\hline Pretty & 35 & 32 & 3 & 1.09 \\
\hline $\begin{array}{l}\text { Woman/Female/Women } \\
\text { or Man/Male/Men }\end{array}$ & 14378 & 13208 & 1170 & 1.09 \\
\hline Cool & 16 & 15 & 1 & 1.07 \\
\hline Great & 538 & 509 & 29 & 1.06 \\
\hline Success & 151 & 144 & 7 & 1.05 \\
\hline Simply & 25 & 24 & 1 & 1.04 \\
\hline Luck & 273 & 272 & 1 & 1.00 \\
\hline Convincingly & 2 & 2 & 0 & 1.00 \\
\hline Prodigy & 10 & 11 & -1 & 0.91 \\
\hline Win & 4386 & 5299 & -913 & 0.83 \\
\hline Hero & 28 & 34 & -6 & 0.82 \\
\hline Calm & 5 & 7 & -2 & 0.71 \\
\hline Bronze & 2126 & 3003 & -877 & 0.71 \\
\hline Settle & 126 & 182 & -56 & 0.69 \\
\hline Talent & 46 & 72 & -26 & 0.64 \\
\hline Young & 180 & 309 & -129 & 0.58 \\
\hline Disgrace & 4 & 28 & -24 & 0.14 \\
\hline Glamor/Glamorous & 0 & 1 & -1 & 0.00 \\
\hline Attractive & 0 & 5 & -5 & 0.00 \\
\hline Ladies/Lady & 31 & 0 & 31 & - \\
\hline Cute & 2 & 0 & 2 & - \\
\hline Feminine & 0 & 0 & 0 & - \\
\hline
\end{tabular}

Note. *Frequency of word appears in tweets about female athletes minus those in male athlete tweets.

**Frequency of word appears in tweets about female athletes divided by those in male athlete tweets. 